Khaja M, et al. Ann Case Report 6: 718.

www.doi.org/10.29011/2574-7754.100718

www.gavinpublishers.com

Case Series

GAVIN PUBLISHERS

\title{
Central Line: Common Bedside Procedure
}

\section{Misbahuddin Khaja ${ }^{1^{*}}$, Alla Yugay ${ }^{2}$, Shoaib Ashraf ${ }^{3}$}

${ }^{1}$ Attending Physician, Division of Pulmonary and Critical Care Medicine, Bronx Care Health System, Affiliated with Icahn School of Medicine at Mount Sinai 1650 Grand Concourse, Bronx, NY 10457, USA

${ }^{2}$ Second Year Pulmonary Fellow, Division of Pulmonary and Critical Care Medicine, Bronx Care Health System, Affiliated with Icahn School of Medicine at Mount Sinai 1650 Grand Concourse Bronx, NY 10457, USA

${ }^{3}$ Third Year Medical Resident, Department of Medicine, Bronx Care Health System, Affiliated with Icahn School of Medicine at Mount Sinai 1650 Grand Concourse Bronx, NY 10457, USA

*Corresponding author: Misbahuddin Khaja, Attending Physician, Division of Pulmonary and Critical Care Medicine, Bronx Care Health System, Affiliated with Icahn School of Medicine at Mount Sinai 1650 Grand Concourse, Bronx, NY 10457, USA.

Citation: Khaja M, Yugay A, Ashraf S (2021) Central Line: Common Bedside Procedure. Ann Case Report 6: 718. DOI: 10.29011/2574-7754.100718.

Received Date: 20 December 2021; Accepted Date: 27 December 2021; Published Date: 30 December 2021

\begin{abstract}
The central line venous catheter is a large bore catheter that has multifunctional use. It is placed with ultrasound guidance with sterile technique. In this review, we discuss the usage of ultrasound for central line placement to improve safety, quality of the procedure, technique, and indication. The common indications for placement include infusion of pressure, fluids, blood products, and emergency access. Ultrasound is used to visualize the anatomy of the vein, location to the nearby artery, and its patency. It gives the real-time image of the vein being punctured by a needle, the position of the guidewire, and finally confirms the position of the catheter. Ultrasound guidance minimizes common complications like placing a catheter in the artery, laceration, and loss of guidewire and pneumothorax. Though the central line insertion is done by a certified healthcare team, including medical residents, fellows, and attending physicians, its maintenance is done by the nursing team.
\end{abstract}

\section{Introduction}

A Central venous catheter is a large bore catheter placed in sterile technique under ultrasound guidance. Central venous lines are used in medical, surgical intensive care units, hematology, and oncology services. In addition, central venous catheters are used for fluid resuscitation, use of vasoactive medications, for placement of swan Ganz catheter, temporary trans venous pacer wires, and hemodialysis [1].

Seldinger, in 1953 described a simple technique of placement of central line under ultrasound guidance. The use of ultrasound has been recommended by the National Institute for Clinical Excellence (NICE). The use of ultrasound reduces complications related to the procedure [2].

Central venous catheters are placed in three large veins femoral vein, internal jugular vein, and subclavian vein. Ultrasound helps to localize the vein in relation to the artery [3].

\section{Indications}

1. The common indications for central line placement are $[4,5]$;

2. Fluid resuscitation.

3. Need for vasopressors in hemodynamically unstable patients.

4. Infusion of blood products during massive transfusion protocol.

5. Catheter needed for Hemodialysis.

6. For infusion for hyperosmolar agents like 3\% hypertonic saline.

7. Infusion of drugs, which can lead to phlebitis if used through peripheral vein access.

8. Emergency venous access during resuscitating cardiac arrest patient.

9. Central venous pressure monitoring. 
10. Placement of pulmonary artery catheter.

11. For placing of Trans venous pacing wire.

\section{Contraindications}

The contraindication for placement of central venous catheter includes [6,7];

1. Infection at insertion site including cellulitis.

2. No compressible vein with visible thrombus in the vein.

3. Distorted Anatomy.

4. Artery is above the vein.

5. Suspected vascular injury, trauma to the local site like cervical spine injury with a cervical collar, trauma to pelvis with pelvic binders.

\section{Coagulopathy.}

\section{Central line kit contents}

Most central line kits have sterile gloves, gown, cap, facemask, shield, saline flush, antiseptic cleaning agent chlorhexidine, and dressing to cover the insertion site. The other part of the kit has $1 \%$ lidocaine for local anesthesia, needle, syringe, introducer needle, guidewire, scalpel, dilator, triple lumen, double lumen catheter, sterile dressing with the suture needle. The vascular ultrasound is equipped with sterile gel and probe.

\section{Insertion Technique}

Place the patient in reverse Trendelenburg position with head turned to the opposite side for the internal jugular, subclavian vein access. For femoral vein access, expose the inguinal area with the knee flexed. After cleaning with chlorhexidine, cover with a sterile drape.

Use ultrasound to differentiate between artery and vein. The use of real-time ultrasound is necessary to check the patency of the central vein. If the vein is compressible, then there is no obstruction. Also, check for visible thrombus in the vein. Give 1\% lidocaine for local anesthesia. Advance introducer needle toward vein by direct visualization by using ultrasound. Once the needle is in the vein, aspirate venous blood, remove syringe and advance guidewire, then remove introducer needle. Again, use ultrasound to confirm the guidewire position in vein both horizontally and longitudinal view. Make a small stab with the scalpel tip, and then use a dilator to dilate the site by twisting gently. Then insert central line over a guidewire and as you advance central line, with drawl guidewire. Make sure to remove the guidewire altogether. The next step is to flush all ports of the central line. Finally, suture the line and apply a sterile dressing.

Confirmation can be done by getting chest radiography and by bubble study using ultrasound. Check for seashore signs to exclude pneumothorax. In addition, the operator should get familiar with stratosphere signs with significance for pneumothorax.

\section{Complications}

Although generally considered a low-risk intervention, we do come across complications of thoracentesis $[8,9]$.

1. Hematoma

2. Bleeding

3. Arterial Puncture

4. Injury to the Nerve

5. Infection

6. Pneumothorax

7. Missing Guidewire

8. Arrhythmias

9. Air Embolism

\section{Conclusion}

Central line insertion using ultrasound has become a part of the procedural training of medical, surgical residents, and fellows. During the training, they are taught how to insert lines, identify complications, and maintain sterility to prevent central line infections.

\section{Acknowledgments}

No funding was provided for the production of this case paper.

\section{Disclosures}

None of the authors has a financial relationship with a commercial entity that has an interest in the subject of the manuscript. No financial support was used for this case series.

\section{Authors Contributions}

M. Khaja, Yugay and Ashraf searched the literature and wrote the manuscript. M. Khaja conceived and edited the manuscript. All authors have made significant contributions to the manuscript and have reviewed it before submission. All authors have confirmed that the manuscript is not under consideration for review at any other Journal. All authors have read and approved the final manuscript.

\section{References}

1. Björkander M, Bentzer P, Schött U, Broman ME, Kander T (2019). Mechanical complications of central venous catheter insertions: A retrospective multicenter study of incidence and risks. Acta Anaesthesiol Scand. 63: 61-68. 
Citation: Khaja M, Yugay A, Ashraf S (2021) Central Line: Common Bedside Procedure. Ann Case Report 6: 718. DOI: 10.29011/25747754.100718.

2. Peter Korsten, Eirini Mavropoulou, Susanne Wienbeck, David Ellenberger, Daniel Patschan (2018) et al. The "rapid atrial swirl sign" for assessing central venous catheters: Performance by medical residents after limited training. PLoS One. 13: e0199345.

3. Rivard AB, Kortz MW, Burns B (2021). Anatomy, Head and Neck, Internal Jugular Vein. StatPearls Publishing.

4. Saugel B, Scheeren TWL, Teboul JL (2017). Ultrasound-guided central venous catheter placement: a structured review and recommendations for clinical practice. Crit Care. 21: 225.

5. Leib AD, England BS, Kiel J (2020). Central Line. StatPearls Publishing.

6. Smith RN, Nolan JP (2013). Central venous catheters. BMJ. 347: f6570.

7. Smith RN, Nolan JP (2013). Central venous catheters. BMJ. 347: f6570.

8. Kashif M, Hashmi H, Jadhav P, Khaja M (2016). A Missing Guide Wire After Placement of Peripherally Inserted Central Venous Catheter. Am J Case Rep. 17: 925-928.

9. Mishra R, Reddy P, Khaja M (2016). Fatal Cerebral Air Embolism: A Case Series and Literature Review. Case Rep Crit Care. 2016: 3425321. 